\title{
Validation of the Systemic Clinical Outcome and Routine Evaluation (SCORE-15) self-report questionnaire: index of family functioning and change in Swedish families
}

Maria Zetterqvist, H. Erneroth Hanell, Marie Wadsby, Madeleine Cocozza and Per Gustafsson

The self-archived postprint version of this journal article is available at Linköping University Institutional Repository (DiVA):

http://urn.kb.se/resolve?urn=urn:nbn:se:liu:diva-163364

N.B.: When citing this work, cite the original publication.

Zetterqvist, M., Hanell, H. E., Wadsby, M., Cocozza, M., Gustafsson, P., (2020), Validation of the Systemic Clinical Outcome and Routine Evaluation (SCORE-15) self-report questionnaire: index of family functioning and change in Swedish families, Journal of Family Therapy, 42(1), 129-148. https://doi.org/10.1111/1467-6427.12255

Original publication available at:

https://doi.org/10.1111/1467-6427.12255

Copyright: Wiley (12 months)

http://eu.wiley.com/WileyCDA/ 
Validation of the Systemic Clinical Outcome and Routine Evaluation (SCORE-15) Self-Report Questionnaire: Index of Family Functioning and Change in Swedish Families 
Instruments for evaluating progress and outcome of systemic therapeutic treatments in clinical practice need to be easily administered and have sound psychometric properties. The Systemic Clinical Outcome and Routine Evaluation, 15-item version (SCORE-15), is a selfreport questionnaire that measures aspects of family functioning. This study investigates the psychometric qualities of a Swedish version of SCORE-15. Seventy Swedish families with healthy children and 159 families with children with psychiatric or behavioural problems were included in the study, resulting in a total of 397 individuals. Results showed that SCORE-15 differentiated clinical from non-clinical families with acceptable psychometric properties for test-retest, internal consistency, convergent and construct validity, as well as sensitivity to change for the clinical sample. The three-factor solution of strengths, difficulties and communication was tested. Results imply preliminary psychometric support for the use of the Swedish version of SCORE-15 to evaluate progress and outcome in clinical practice. Keywords: SCORE-15, self-report questionnaire, outcome measure, validation, factor analysis, reliability.

Running head: Validation of SCORE-15

Practitioner points

- SCORE-15 is an easily administered questionnaire suitable for use in clinical practice to evaluate systemic therapeutic progress and outcome.

- The Swedish version of SCORE-15 has acceptable psychometric properties. 


\section{Introduction}

As in most other European countries, there has been a paucity of outcome measures of family and systemic therapeutic services in Sweden, regrettably hampering evaluations of such interventions. Although there are quantitative self-report outcome measures in family therapy treatment research (e.g., Family Assessment Device [FAD; Epstein et al., 1983]; Family Adaptability and Cohesion Evaluation Scales [FACES-IV; Olson, 2011] and The Family Assessment Measure [FAM III; Skinner et al., 2000]), they are still not used routinely in clinical practice. The implementation of such routines can be challenging for clinicians, who need quick and easily administered instruments suitable for children, adolescents and also adults in the family, and at the same time they have to take into account the instrument's psychometric properties and be aware of the pitfalls involved when selecting instruments.

The advantages of measuring progress and outcome are several and the use of such instruments needs to be encouraged (Stratton, 2017; Wilkinson, 1987), but it is not without challenges (Moran, 2017). Whether an instrument will be used and spread by clinicians depends largely on its meaningfulness, i.e. that it measures aspects that are considered crucial, and that it is easily administered (Moran, 2017). In addition to other psychometric properties, an instrument's sensitivity to change is central to the purpose of evaluating therapeutic interventions (Vermeersch et al., 2000).

Furthermore, there are issues concerning the appropriateness of the measures mentioned above. In a systematic review conducted in 2016 (Hamilton and Carr, 2016), the authors commented that these scales were created decades ago (with the exception of the revised FACES-IV measure) and only a minority of the studies identified were conducted in the last 10 years. The measures were designed for use within the framework of their respective theoretical models of family functioning. Therefore, they may not represent current theoretical, therapeutic, or clinical practices. For example, older measures and validation 
studies may not reflect the current movement in the field of psychotherapy towards an integrative approach, which has been documented by Breunlin et al. (2011). Hamilton and Carr (2016) concluded that "The systemic clinical outcome and routine evaluation (SCORE) stands out as a new and particularly useful scale for routine use in clinical practice" (page 27).

The SCORE-15 (Stratton et al., 2010) questionnaire measures families' functions and changes and is an established measurement that has been available in English since 2010. During the last decade it has been spread within the UK and to several other countries, such as Portugal and Poland (e.g., Cahill et al., 2010; Fay et al., 2013; Hamilton et al., 2015; Józefik et al., 2015; Teh et al., 2017; Vilaca et al., 2015). At present it is available in 22 languages. A 15-item version was developed from the original 40-item version, which in its turn was inspired by Clinical Outcomes in Routine Evaluation (CORE; Barkham et al., 1998, Evans et al., 2000). A systemic perspective was added to measure change in relationships with the explicit purpose of being easy to use in clinical practice. SCORE-15 offers users short descriptions of various aspects of family interaction that have proven important for families in therapy. It can be used to supervise and report established progress in systemic family therapy. Since SCORE-15 identifies significant questions relating to a family's interactions, the instrument has many potential uses in therapy. It is easy to administer, and it evaluates changes in family function over time (Carr and Stratton, 2017; Hamilton and Carr, 2016).

SCORE-15 has shown to be potentially promising in measuring therapy results and therapeutic changes in the family (Stratton et al., 2014). The 15-item version, besides being quick and easy to administer, has the additional advantage that it can be used for both children and adults (Jewell et al., 2013). Strengthening it further, the UK Association for Family Therapy (AFT) and the European Family Therapy Association (EFTA) have selected 
SCORE as the main instrument for measuring therapeutic outcome in systemic family therapy (Carr and Stratton, 2017).

SCORE-15 is thus considered a welcome addition to the measurement of outcome in systemic treatment and research. Sound psychometric properties are essential if an instrument is to be widely spread. Psychometric research on SCORE is emerging towards this aim, but as Carr and Stratton (2017) pointed out in their recent review, previous studies on SCORE are not without limitations. Examples of such limitations are small samples and large drop-out in outcome measurements. Other studies lack test-retest or do not include factorial analysis in their validity testing. These are issues that need to be addressed in order for SCORE to be spread more widely and fill the gap in systemic outcome measures outside the UK.

The aim of the present study is to investigate the psychometric qualities of a Swedish version of SCORE-15, addressing most of the shortcomings reported by Carr and Stratton (2017). We investigate the reliability and validity of SCORE-15 in a sample of Swedish families. The main purpose is to examine the instrument's usefulness in differentiating between clinical and non-clinical families; whether the earlier underlying constructs of the three factors of strengths, difficulties and communication can be replicated; and whether the instrument correlates with similar constructs and is sensitive to change, as well as being reliable over time.

\section{Methods}

\section{Participants}

Seventy families with healthy children and 159 families with children with psychiatric or behavioural problems, for which they were in contact with child psychiatry or social services, were included in the study. This resulted in a total of 397 participants. Of these, 316 were 
parents (215 clinical and 101 non-clinical) and 81 were children or adolescents (45 clinical and 36 non-clinical).

\section{Procedure}

Non-clinical participants. Non-clinical participants were recruited from April to September 2016 from small and large Swedish cities in the counties of Stockholm, Östergötland and Kronoberg. For recruitment of the normative sample, eight schools, three preschools and one city council child care centre were contacted. Of these 12 eligible institutions, 7 agreed to participate. Nurses at the child care centre gave SCORE-15 to parents and answers were sent in by post. Preschool teachers gave the questionnaire to parents, who returned them to the staff. In one school, one of the authors distributed SCORE-15 to school classes and the completed questionnaires were then collected by teachers. In two of the other schools, students were given information about the study by class teachers and parents were informed on the school webpage before the material was sent home by post together with a response envelope. A total of 137 questionnaires were returned. Of these, 101 were completed by adults and 36 by children and adolescents, aged $10-17$ years with a mean age of 13.8 years.

Clinical participants. Clinical participants were recruited from three child and adolescent psychiatric outpatient clinics and two family treatment units in the social services in four middle to large-sized towns in the counties of Sörmland, Uppsala, Örebro and Östergötland in Sweden.

Social services. All the families who were in treatment for the first time for communication and relationship problems at two family treatment units during the time period from September 2015 to March 2016 were consecutively asked to participate. A total of 34 families participated, which resulted in 71 answered SCORE-15 questionnaires. Of these, 50 were filled in by adults and 21 by children and adolescents from the age of 11 to 18 years with a mean age of 14.4 years. Each family member completed SCORE-15 and the 
Swedish self-report questionnaire Family Climate (Hansson 1989, Skerfving et al., 2014) twice: before family treatment and at a follow-up after the completed course of treatment, or three months after treatment started. Of the 34 families where SCORE-15 questionnaires filled in by adults were available, $23(67.6 \%)$ participated in the follow-up. The same members of each family completed the measures before treatment and at the follow-up.

Child and adolescent psychiatric clinics. All the families who were in contact with the child and adolescent psychiatric clinic in the town of Linköping for their first assessment during a seven-month period from March 2016 to September 2016 were routinely asked by administrative staff to participate in the study: If families agreed to participate they were given SCORE-15 and the Strengths and Difficulties Questionnaire (SDQ), together with some other measures not used in the current study. A total of 61 SCORE-15 questionnaires from 37 families (36 parents and 25 children and adolescents from the age of 11 years and older, mean age 13.8) were collected.

Parents of children who had recently been diagnosed with Attention Deficit Hyperactivity Disorder (ADHD) and who participated in a parental psychoeducational training programme at two child and adolescent clinics during 2015-2016 in the towns of Nyköping and Örebro were asked during the first session to participate in the study. A total of 88 out of 115 eligible families (76.5\%) agreed to participate, resulting in 128 questionnaires completed by parents.

\section{Measures}

The Systemic Clinical Outcome and Routine Evaluation, 15-item version (SCORE-15; Stratton et al., 2010), is a self-report instrument measuring family processes and aspects of family functioning. It is mainly used for family members 11 years and older. Its 15 items are rated on a five-point Likert scale, from 1 "describes my family - extremely well” to 5 “describes my family - not at all”. Total score ranges from 15 to 75, where high scores 
indicate more family problems. Respondents are then asked to rate perceived burden of problems and family functioning on three 10-point Likert scales. SCORE-15 takes approximately 5-10 minutes to administer. An overall sum of family adjustment is obtained, as well as results on three factors: "strengths"; "We are good at finding new ways to deal with things that are difficult", "difficulties"; "We seem to go from one crisis to another in my family" and "communication"; "It feels risky to disagree in our family". In a recent review (Carr and Stratton, 2017), SCORE-15 was shown to have sound psychometric qualities. It has been used on adolescents and adults in both clinical and non-clinical samples. Good reliability has been found with test-retest coefficients between .81-.94 for total scores and the three subscales and Cronbach's alpha ranging between .78-.93 for the total scale in different studies. Cronbach's alpha for the three subscales ranged from .59 to .90 (Carr and Stratton, 2017). There were also significant correlations with child mental health difficulties and life satisfaction, supporting construct validity (Fay et al., 2013; Hamilton et al., 2015; O'Hanrahan et al., 2017; Jewell et al., 2013; Stratton et al., 2014; Teh et al. 2017). Good reliability with alpha ranging from $.80-.93$ for the total scale, but poor to fair alpha for the subscale communication has been found (e.g., Fay et al., 2013; Hamilton et al., 2015; O'Hanrahan et al., 2017; Stratton et al., 2014; Teh et al., 2017).

Translation protocol. For the translation procedure of the present study, the authors were in close collaboration with the UK SCORE researchers Prof emeritus Peter Stratton, Dr Renee Singh and Judith Lask to handle linguistic and cultural issues and adaptability to ensure that the measure can show cross-cultural comparability. Together with the UK collaborators the SCORE Translation protocol Jan2014 was followed and a Swedish Translation protocol was made that was agreed on by the UK collaborators. The SCORE translation protocols are submitted as supplements. 
The Strengths and Difficulties Questionnaire (SDQ; Goodman et al., 1998) is used extensively worldwide. It measures children's (5-15 years) behaviours in 25 items. It can be used by parents, teachers and children from the age of 10 . The items are divided into five subscales with five items in each: emotional symptoms, conduct problems, hyperactivity/inattention, peer problems and prosocial behaviours. There are also additional questions on functional impairment at home, in school and with friends (not used in this study). Earlier psychometric studies have also shown good psychometric properties (e.g., Ortuno-Sierra et al., 2015) in a Swedish context (Gustafsson et al., 2016; Smedje et al., 1999) where it has been shown to differentiate clinical from non-clinical groups (Malmberg et al., 2009) and is considered a valuable instrument for screening children and adolescents. It was translated into Swedish by Smedje and colleagues and its psychometric properties were investigated in a sample of 900 children (Smedje et al., 1999). After a first translation, the authors used the SDQ in 12 clinical child psychiatric cases, rated by parents and teachers. The questionnaire was found to be easy to use in a clinical setting with adequate face validity. Clinicians in child psychiatry also rated their own children according to the SDQ and were also interviewed. After revisions, the final translation was retranslated into English by an independent translator with a satisfactory result. An informal test-retest procedure was performed on 15 children, showing a Spearman rank order correlation of .96 on the total score over a two-week period (Smedje et al., 1999).

Familjeklimat (= Family Climate) is a Swedish family diagnostic measure of the emotional climate in the family developed by Hansson (1989). It can be used by adults and children from the age of 11 years. The instrument consists of 85 adjectives, yielding a description of how family members perceive the emotional climate in the family. The instrument was developed to suit clinical praxis. It can be subdivided into four dimensions: "Closeness" includes 18 adjectives describing a positive climate with warmth, security and harmony; 
"Distance" comprises 11 adjectives describing a negative atmosphere characterised by coolness and rejection; "Spontaneity" relates to six adjectives describing the family's emotional expressiveness in both positive and less positive terms; "Chaos" pertains to six adjectives that relate to a state of disorder within the family. Each family member should mark a total of at least 15 adjectives that describe their family. It has previously been used to evaluate family interventions (Skerfving et al., 2014). Construct validity was shown to be satisfactory as was test-retest reliability, $\mathrm{r}=.91$ after 3 weeks and $\mathrm{r}=.86$ after 5 months (Hansson, 1989; Söderlind and Johansson, 2004), although samples have been small. The subscale spontaneity was not used in the present study, since some validity issues have been raised concerning this subscale (Söderlind and Johansson, 2004).

\section{Ethics}

A potential ethical dilemma could arise if completed questionnaires brought to light children with behavioural problems that had not been formally identified, i.e. custodians were not aware of it. However, in such cases the research group was prepared to provide relevant forms of help. The study was approved by the Regional Ethical Review Board of Linköping (Dnr 2015/347-31).

\section{Data Analysis}

Descriptive statistics were analysed using frequencies and percentages, mean values and standard deviations, with cross-tabulation using chi-square $\left(\chi^{2}\right)$ and paired samples t-test for comparing mean value differences within groups, and two-sided t-test was used between groups. Results were also run using non-parametric Mann-Whitney analysis for between group comparisons and Wilcoxon signed ranks test for within group comparisons. Effect size (ES) was calculated using Cohen's (1988) criteria of .20, .50 and .80 for small, medium and large effect. For test-retest reliability and concurrent validity, Pearson's product-moment 
correlation coefficient $(r)$ was used. Internal consistency was assessed using Cronbach's alpha $(\alpha)$. If more than one item within the same factor on SCORE-15, or more than two of the total 15 items were missing, the result for that person was omitted from further analyses, since imputing two items in a factor consisting of five items would violate the robustness of the findings. This was the case with two questionnaires. For individuals with fewer missing items, imputation with the mean score on that factor for that individual was regarded as trustworthy. This was the case for 19 individuals $(4.8 \%, n=397)$. All the analyses were done both with and without imputation with only minimal differences. This was also the case for internal consistency and structural analysis, where imputation can potentially affect results._All the results reported throughout are with imputation, except for internal consistency. A confirmatory factor analysis (CFA) was performed on the clinical group of parents using AMOS 24.0 (SPSS Inc, Chicago, IL) with maximum likelihood estimation method and following recommendations for non-significant $\chi^{2}$, model fit of minimum $\geq .90$ for incremental fit index (IFI), comparative fit index (CFI), Tucker Lewis index (TLI) $\geq .90$, and root-mean-square error of approximation (RMSEA) <.08 (as in Hamilton et al., 2015), and

$\chi^{2} / d f \leq 2$, and also with normed fit index $(\mathrm{NFI}) \geq .90$. The factors were allowed to correlate, as in Hamilton et al. (2015). In order to deal with potential issues of non-independence, where different members of each family rate the same family, which can affect structural analysis, the CFA was also re-run using family mean scores. All other statistical analyses were performed using the SPSS 24.0 software package.

\section{Results}

There were significantly more married and co-habiting parents in the non-clinical group, and more single parents in the clinical group $\left(\chi^{2}=35.42, p<.001\right)$. Significantly more parents in 
the non-clinical group also had a college/university degree compared to the clinical parents $\left(\chi^{2}\right.$ $=11.82, p=.008)$. Sample characteristics are provided in Table 1 .

\section{Reliability}

Test-retest. Twenty-two participants (of which 16 were mother and father from the same family) from the non-clinical sample filled in SCORE-15 on two occasions 1-2 weeks apart. Correlations between measurement 1 and 2 were .81 (95\% CI .66-.91) for the total scale, .56 (95\% CI .20-.81) for strengths, .80 (95\% CI .57-.93) for difficulties and .69 (95\% CI .47-.87) for communication ( $p<.001$ for all comparisons). Results indicate moderate test-retest reliability for this sample.

Internal consistency. Cronbach's alpha for SCORE-15 for the adults in families with clinical contact ( $n=204$ without imputation) was .82 for difficulties, .73 for strengths and .69 for communication, indicating acceptable internal reliability. Cronbach's alpha was also calculated based on family mean values for clinical families $(n=151$ without imputation), resulting in $\alpha=.82, .75$ and .71 , respectively. Internal consistency for healthy families ( $n=67$, family mean average without imputation) was not quite adequate with $\alpha=$ .57 for difficulties, .68 for strengths and .53 for communication.

\section{Validity}

Examination of SCORE-15 data show that kurtosis and skewness did not exceed +/-1.0 on SCORE total data, or on the three subscales for the total sample of $n=397$. Data for clinical adults ( $n=215)$, on which the CFA is based, were also broadly normally distributed, and the same was true for clinical children. However, for non-clinical adults and children the data violated normal distribution. Differences between and within groups in Table 2 and Table 3 were therefore checked with non-parametric tests, which did not affect results. 
Criterion validity. There were significant differences between mean average scores on both the total SCORE-15 score and the subscales strengths, difficulties and communication between clinical and non-clinical parents, as well as clinical and non-clinical children and adolescents $(p<.001$ for all comparisons except for communication in adolescents, $p=.021$ ). The clinical groups reported higher overall scores (Table 2). Results were confirmed using non-parametric tests.

Convergent and discriminant validity. Thirty-six parents whose children were in contact with a child and adolescent psychiatric clinic, answered both SCORE-15 and SDQ about the index patient children $(n=36)$ in the family. There was a significant correlation between the total SDQ score and the total SCORE-15 score $(r=.42, p<.05)$, and for family difficulties $(r=.42, p<.01)$ and communication $(r=.47, p<.01)$, but not for strengths. Some SDQ subscales correlated more strongly with the SCORE-15 than others. The emotional subscale had no significant correlation with SCORE, whilst SDQ conduct problems more clearly correlated with both the total SCORE-15 score $(r=.22, p<.01)$ and family difficulties $(r=.54, p<.01)$ and problems with communication $(r=.49, p<.01)$. SDQ Hyperactivity was also related to family difficulties $(r=.33, p<.05)$ and communication $(r=.36, p<.05)$. Adolescent peer problems were related to total SCORE-15 scores $(r=.35, p<.05)$ and communication $(r=.43, p<.01)$, but not to strengths or difficulties. There was also support for discriminant validity, with SDQ prosocial behaviours negatively correlating with total scores on SCORE-15 $(r=-.47, p<.01)$ strengths $(r=-.40, p<.05)$, difficulties $(r=-.49, p<$ $.01)$ and communication $(r=-.39, p<.05)$. Applying Bonferroni correction for the number of tests (24), none of the above correlations are statistically significant. Thus no real conclusions can be drawn about the convergent validity between the SDQ and SCORE when rating individual children until larger samples are available. Results can rather be interpreted as supporting some convergence of results on SDQ and SCORE-15 and that parents who attend 
child psychiatry show ratings of their family's functioning which broadly correlate with their ratings of the child's state.

In the clinical group, 179 parents (from 123 families) filled in both SCORE-15 and the questionnaire Family Climate. There were significant correlations (all three $p \leq .01$, i.e. below the Bonferroni corrected criterion $.05 / 3=.0167$ ) between all the subscales on Family Climate and the total average score on SCORE-15. Those with a higher score on SCORE-15 reported less closeness $(r=-.62, p<.01)$, more distance $(r=.44, p<.01)$ and more chaos $(r=.51, p<.01)$, supporting convergent validity.

Sensitivity to change. Results for total score $(p=.009)$ and subscale strengths on SCORE-15 (filled in by adults) decreased significantly $(p=.001)$ between time 1 and time 2 following a three-month family treatment intervention for 23 clinical families (Table 3 ). The subscale communication showed a trend $(p=.07)$, whilst the subscale problems did not reach statistical significance $(p=.15)$. Results were also checked using non-parametric Wilcoxon signed ranks test with similar findings. Effect sizes, however, were small.

Construct validity. All indicator variables loaded significantly on the latent factors $(p<.001)$ in the CFA of the original three-factor model and it resulted in an acceptable fit in this sample of clinical adults $\left(\chi^{2}(87, N=215)=172.37, p<.001, \mathrm{CMIN} / \mathrm{df}=\right.$ $1.98, \mathrm{NFI}=.84, \mathrm{IFI}=.91, \mathrm{TLI}=.89, \mathrm{CFI}=.91, \mathrm{RMSEA}=.068(90 \% \mathrm{CI}[.053, .082]))$. The

standardised factor loadings and factor correlations are presented in Table 4. Similar model fit indices with close to acceptable fit $\left(\chi^{2}(87, N=159)=193.66, p<.001, \mathrm{CMIN} / \mathrm{df}=2.23, \mathrm{NFI}\right.$ $=.79, \mathrm{IFI}=.87, \mathrm{TLI}=.82, \mathrm{CFI}=.87, \mathrm{RMSEA}=.069(90 \% \mathrm{CI}[.056, .082]))$ were reached using family mean scores for members of 159 clinical families (See Table 4).

\section{Discussion}

This study addresses the need in clinical practice of family therapy for updated and new quantitative outcome research instruments in Sweden by psychometrically evaluating 
SCORE-15 in a Swedish context. SCORE-15 delimited clinical from non-clinical groups, for both children/adolescents and parents, with significantly higher results for clinical adults and children than non-clinical.

However, both non-clinical and clinical individuals in our samples reported average scores below the $90^{\text {th }}$ percentile cut-off of found in Fay et al. (2013), which suggests that our samples are not representative of the families with the lowest family functioning in the UK sample. Our results are in line with previous studies on SCORE-15 which have shown to differentiate clinical from non-clinical groups in England, Ireland and Portugal (Fay et al., 2013; Hamilton et al., 2015; O'Hanrahan et al., 2017; Stratton et al., 2014; Vilaca et al., 2015), indicating preliminary support for criterion validity for the Swedish translated version of SCORE-15 compared to UK data.

We also investigated the underlying constructs in SCORE-15. Using CFA, we found acceptable support for factors strengths, difficulties and communication using a sample of parents who were in contact with the social services or child psychiatry for their child's psychiatric or behavioural difficulties. We could not run CFA for the other samples, due to their smaller sample size. Earlier UK data on factor analytic studies on SCORE-15 are somewhat inconsistent, since some studies have replicated the three-factor model (Fay et al., 2013; Hamilton et al., 2015; Stratton et al., 2010), whilst others have not (O’Hanrahan et al., 2017; Stratton et al., 2014). Carr and Stratton (2017) suggest that the underlying dimensions of the instruments are perhaps valid in some populations, but not in others. It is also possible that family function as a concept does not show a clean factorial structure that easily.

With regard to internal consistency for the respective subscales, it was acceptable for the total score and the subscales strengths and difficulties but less satisfactory for the subscale communication, which has also previously been found in UK data by Fay et al. (2013). 
There were significant, but not very high, correlations between SCORE-15 and SDQ, and between SCORE-15 and Family Climate, confirming that SCORE-15 captures psychological ill-health measured both on an individual level and on a family level. It seems reasonable that the associations between SCORE-15 and SDQ were moderate, since individual problems differ in certain respects from family problems. There are some previous UK studies comparing SDQ and SCORE-15 with similar findings (e.g., Fay et al., 2013; Hamilton et al., 2015). The SDQ subscale Emotional Problems did not correlate with selfreported family difficulties, communication or strengths, which points to the possibility that internalising behaviours either do not affect family relations or are perceived as individual problems. On the other hand, the SDQ subscale Conduct Problems correlated significantly with the total SCORE-15 score and all subscales, supporting the instrument's concurrent and discriminant validity and strengthening the relationship between family dysfunction and conduct disorder (Button et al., 2005). Hypothetically, the items in SCORE-15 capture aspects such as harsh family emotional climate, negative parental emotional expressivity, lack of positive parenting and communication difficulties that have been associated with the development of externalising behaviour (Farrington, 2005). The number of ratings with both SCORE-15 and SDQ was small, however, so interpretations must be tentative. Moreover, interpretations of convergent validity are complicated by the fact that SDQ rates individuals whilst SCORE rates families, which needs to be taken into account.

Scores on SCORE-15 decreased significantly after a three-month family intervention in the social services, potentially indicating sensitivity to change. Effect sizes were small, however, which is in line with previous UK data, where a significant, but relatively small, reduction of total SCORE-15 scores have been shown following family interventions (Hamilton et al., 2015). There are, however, also some contradictory results, 
which indicate less responsiveness to change following three family intervention sessions in a Polish sample by Jozefik et al. (2015).

\section{Limitations}

This study contributes important information to the aim of increasing easily administered and clinically meaningful and psychometrically sound instruments in the field of family therapy. It is not, however, without limitations. One of the main drawbacks is that there is missing information on how many were eligible of those who participated, and how many declined participation at the two family treatment units and the three child and adolescent psychiatric clinics, which means that information is lacking on drop-out rates. This is also the case for the non-clinical data. This means that caution has to be taken when discussing generalisability and representativeness of our data. A formal a priori statistical power/sample size calculation was not done, which is a limitation. However, in order to perform CFA, we aimed for at least 10-15 participants per variable. With 15 items in the SCORE-15, this would mean 150-225 adult clinical participants. Data collection was finished when the allotted time period of 6-7 months to collect data had passed. 215 adult clinical participants had then been recruited. The investigated non-clinical families responded to a non-specific invitation, and it is quite possible that they reflect families with a special interest in family relations. For the clinical samples we have no information on non-participants. In addition, the educational level and proportion of co-habiting parents were higher in the non-clinical families, which could have influenced the ratings. Thus, generalisability could be questioned. The number of participants in the social services family intervention was low and the number of treatment sessions is lacking.

To conclude, results showed that the Swedish version of SCORE-15 differentiated clinical from non-clinical families with acceptable psychometric properties for 
test-retest, internal consistency, convergent and construct validity, as well as sensitivity to change for the clinical sample, lending preliminary support to its clinical meaningfulness and acceptable psychometric qualities in a clinical sample when compared to UK data on validity and reliability. The use of SCORE-15 makes it possible to continually monitor progress (or non-progress) in family therapy, and clinicians could be encouraged to use it in routine care. The Swedish translation of SCORE-15 can be downloaded at http://www.aft.org.uk/view/15101224fle.html

\section{Acknowledgements}

The authors wish to thank all participating families and Katarina Berzell, Monica Nordlander, Ann-Helen Karhatsu, Yvonne Sturesson-Ljungblad, Anna Thurfjell Klein and Camilla Åhlander for the data collection. Also, special thanks to Dr Örjan Dahlström for statistical input. 


\section{References}

Barkham, M., Evans, C., Margison, M., McGrath, G., Mellor-Clarke, J., Milne, D., \& Connell, J. (1998). The rationale for developing and implementing CORE outcome batteries for routine use in service settings and psychotherapy outcome research. Journal of Mental Health, 7: 35-47.

Breunlin, D., Pinsof, W., Russell, W., \& Lebow, J. (2011). Integrative problem centered metaframeworks (IPCM) therapy I: Core concepts and hypothesizing. Family Process, 50: 293-313.

Button, T.M.M., Scourfield, J., Martin, N., Purcell, S., \& McGuffin, P. Family dysfunction interacts with genes in the causation of antisocial symptoms. (2005). Behavior Gentics, 35: $115-120$.

Cahill, P., O’Reilly, K., Carr, A., Dooley, B., \& Stratton, P. (2010). Validation of a 28-item version of the Systemic Clinical Outcome and Routine Evaluation in an Irish context: The SCORE-28. Journal of Family Therapy, 32: 210-231.

Carr, A. \& Stratton, P. (2017). The Score Family Assessment Questionnaire: A Decade of Progress. Family Process, 56: 285-301.

Cohen, J. (1988). Statistical power analysis for the behavioral sciences (2nd ed.). Hillsdale, NJ: Lawrence Erlbaum Associates.

Epstein, N., Baldwin, L., \& Bishop, D. (1983). The McMaster family assessment device. Journal of Marital and Family Therapy, 9: 171-180.

Evans, C., Mellor-Clark, J., Margison, F., Barkham, M., Audin, K., Connell, J., \& Audin, K. (2000). CORE: Clinical Outcomes in Routine Evaluation. Journal of Mental Health, 9: $247-255$. 
Farrington, D. P. (2005). Childhood origins of antisocial behaviour. Clinical Psychology and Psychotherapy, 12: $177-190$.

Fay, D., Carr, A., O’Reilly, K., Cahill, P., Dooley, B., Guerin, S., \& Stratton, P. (2013). Irish norms for the SCORE-15 and 28 from a national telephone survey. Journal of Family Therapy, 35: 24-42.

Goodman, R., Meltzer, H., \& Bailey, V. (1998). The Strengths and Difficulties Questionnaire: a pilot study on the validity of the self-report version. European Child \& Adolescent Psychiatry, 7: 125-130.

Gustafsson, B. M, Gustafsson P. A., \& Proczkowska-Björklund, M. (2016). The Strengths and Difficulties Questionnaire (SDQ) for preschool children - a Swedish validation. Nordic Journal of Psychiatry, 70: 567-574.

Hamilton, E. \& Carr, A. (2016). Systematic Review of Self-Report Family Assessment Measures. Family Process, 55: 16-30.

Hamilton, E., Carr, A., Cahill, P., Cassells, C., \& Hartnett, D. (2015). Psychometric Properties and Responsiveness to Change of 15- and 28-Item Versions of the SCORE: A Family Assessment Questionnaire. Family Process, 54: 454-463.

Hansson, K. (1989). A list of adjectives for family diagnostics. Research report from the Department of Psychology, Number 1, Lund university.

Jewell, T., Carr, A., Stratton, P., Lask, J., \& Eisler, I. (2013). Development of a children's version of the SCORE index of family function and change. Family Process, 52: 673684.

Jozefik, B., Matusiak, F., Wolska, M., \& Ulasiska, R. (2015). Family therapy processWorks on the Polish version of SCORE-15 tool. Psychiatria Polska, www.psychiatriapolska.pl. doi:10.12740/PP/OnlineFirst/42894 
Malmberg, M., Rydell, A. M., \& Smedje, H. (2003). Validity of the Swedish version of the Strengths and Difficulties Questionnaire (SDQ-Swe). Nordic Journal of Psychiatry, 57: $357-363$.

Moran, P. (2017). Selecting self-report outcome measures for use in family and systemic therapy. Journal of Family Therapy, 39: 41-56.

O’Hanrahan, K., White, M. D., Carr, A., Cahill, P., Keenleyside, M., Fitzhenry, M., ... Browne, S. (2017). Validation of 28 and 15 item versions of the score family assessment questionnaire with adult mental health service users. Journal of Family Therapy, 39: 4-20.

Olson, D. (2011). FACES IV and the circumplex model: Validation study. Journal of Marital and Family Therapy, 3: 64-80.

Ortuño-Sierra, J., Chocarro, E., Fonseca-Pedrero, E., Sastre i Riba, S., \&, Muñiz, J. (2015).

The assessment of emotional and behavioural problems: Internal structure of The Strengths and Difficulties Questionnaire. International Journal of Clinical and Health Psychology, 15: 265-273.

Skerfving, A., Johansson, F., \& Elgán, T. H. (2014). Evaluation of support group interventions for children in troubled families: study protocol for a quasi-experimental control group study. BMC Public Health, 14:76 doi:10.1186/1471-2458-14-76

Skinner, H., Steinhauer, P., \& Sitarenios, G. (2000). Family assessment measure (FAM) and process model of family functioning. Journal of Family Functioning, 22: 190-210.

Smedje, H., Broman, J.E., Hetta, J., von Knorring, A.L. (1999). Psychometric properties of a Swedish version of the "Strengths and Difficulties Questionnaire". European Child \& Adolescent Psychiatry, 8: 63-70.

Stratton, P. (2017). Comment on Patricia Moran: 'Selecting self-report outcome measures for use in family and systemic therapy'. Journal of Family Therapy, 39: 57-59. 
Stratton, P., Bland, J. Janes, E., \& Lask, J. (2010). Developing an indicator of family function and a practicable outcome measure for systemic family and couple therapy: the SCORE. Journal of Family Therapy, 32: 232-258.

Stratton, P., Lask, J., Bland, J., Nowotny, E., Evans, C., Singh, R., Janes, E., \& Peppiatt, A. (2014). Detecting therapeutic improvement early in therapy: validation of the SCORE 15 index of family functioning and change. Journal of Family Therapy, 36: 3-19.

Söderlind, M. \& Johnsson, B. (2004). Family climate, a validation. Bachelor thesis, School of Social Work, Lund university.

Teh, Y. Y., Lask, J., \& Stratton, P. (2017). From family to relational SCORE-15: An alternative adult version of a systemic self-report measure for couples and LGB people. Journal of Family Therapy, 39: 21-40.

Vermeersch, D. A., Lamberet, M. J., \& Burlingame, G. A. (2000). Outcome questionnaires: sensitivity to change. Journal of Personality Assessment, 74: 242-261.

Vilaca, M., de Sousa, B., Stratton, P., \& Relvas, A. P. (2015). The 15-item Systemic Clinical Outcome and Routine Evaluation (SCORE-15) scale: Portuguese validation studies. Spanish Journal of Psychology, 18: 1-10.

Wilkinson, I. (1987). Family Assessment: A review. Journal of Family Therapy, 9: 367-380. 


\begin{tabular}{|c|c|c|c|c|}
\hline \multicolumn{5}{|c|}{\begin{tabular}{|l} 
Table 1 \\
Participants' demographics, frequencies and percentages
\end{tabular}} \\
\hline & $\begin{array}{l}\text { Clinical } \\
\text { parents } \\
n=205-215\end{array}$ & $\begin{array}{l}\text { Non-clinical } \\
\text { parents } \\
n=97-101\end{array}$ & $\begin{array}{l}\text { Clinical } \\
\text { children } \\
n=43-45\end{array}$ & $\begin{array}{c}\text { Non-clinical } \\
\text { children } \\
n=36\end{array}$ \\
\hline \multicolumn{5}{|l|}{ Sex } \\
\hline Female & $139(64.7)$ & $63(62.4)$ & $30(66.7)$ & $24(66.7)$ \\
\hline Male & $76(35.3)$ & $38(37.6)$ & $15(33.3)$ & $12(33.3)$ \\
\hline Children's age $(m, S D)$ & & & $14.8(2.03)$ & $13.8(2.01)$ \\
\hline \multicolumn{5}{|l|}{ Ethnicity } \\
\hline Sweden & $193(93.2)$ & $91(93.8)$ & $39(90.7)$ & $35(97.2)$ \\
\hline Europe & $7(3.4)$ & $6(6.2)$ & $0(0.0)$ & $0(0.0)$ \\
\hline Outside Europe & $7(3.4)$ & $0(0.0)$ & $4(9.3)$ & $1(2.8)$ \\
\hline \multicolumn{5}{|l|}{ Parents' family status } \\
\hline Married/co-habitant & $153(71.2)$ & $91(90.1)^{1}$ & & \\
\hline Separated/divorced & $23(10.7)$ & $10(9.9)$ & & \\
\hline Single parent & $39(18.1)$ & $0(0.0)$ & & \\
\hline \multicolumn{5}{|l|}{ Parents' education } \\
\hline University/college & $81(39.5)$ & $60(59.4)^{2}$ & & \\
\hline $\begin{array}{l}\text { High school } \\
\text { (theoretical) }\end{array}$ & $49(23.9)$ & $22(21.8)$ & & \\
\hline $\begin{array}{l}\text { High school } \\
\text { (vocational) }\end{array}$ & $61(29.8)$ & $18(17.8)$ & & \\
\hline Primary school & $14(6.8)$ & $1(1.0)$ & & \\
\hline
\end{tabular}


Table 2 Means, standard deviations, t-test and effect sizes (Cohen's d) for SCORE-15 for clinical and non-clinical samples

\begin{tabular}{|c|c|c|c|c|c|c|c|c|c|c|}
\hline & $\begin{array}{l}\text { Non- } \\
\text { clinical } \\
\text { parents } \\
(n=101)\end{array}$ & $\begin{array}{l}\text { Clinical } \\
\text { parents } \\
(n=215)\end{array}$ & $t$ & $p$ & $\begin{array}{l}\text { Effect size } \\
(95 \% \mathrm{CI})\end{array}$ & $\begin{array}{l}\text { Non- } \\
\text { clinical } \\
\text { children } \\
(n=36)\end{array}$ & $\begin{array}{l}\text { Clinical } \\
\text { children } \\
(n=45)\end{array}$ & $t$ & $p$ & $\begin{array}{l}\text { Effect size } \\
(95 \% \mathrm{CI})\end{array}$ \\
\hline Total average & $1.63(.47)$ & $2.26(.64)$ & 8.90 & $<.001$ & $1.07(.81-1.31)$ & $1.81(.59)$ & $2.40(.77)$ & 3.80 & $<.001$ & $.85(.38-1.30)$ \\
\hline Strengths & $1.69(.51)$ & $2.17(.68)$ & 6.38 & $<.001$ & $.76(.52-1.00)$ & $1.74(.70)$ & $2.45(.95)$ & 3.76 & $<.001$ & $.84(.37-1.29)$ \\
\hline Difficulties & $1.46(.43)$ & $2.46(.89)$ & 10.79 & $<.001$ & $1.29(1.03-1.55)$ & $1.79(.64)$ & $2.50(.95)$ & 3.87 & $<.001$ & $.86(.39-1.31)$ \\
\hline Communication & $1.67(.65)$ & $2.16(.73)$ & 5.94 & $<.001$ & $.71(.42-.99)$ & $1.85(.71)$ & $2.23(.73)$ & 2.35 & .021 & $.53(.08-.97)$ \\
\hline
\end{tabular}




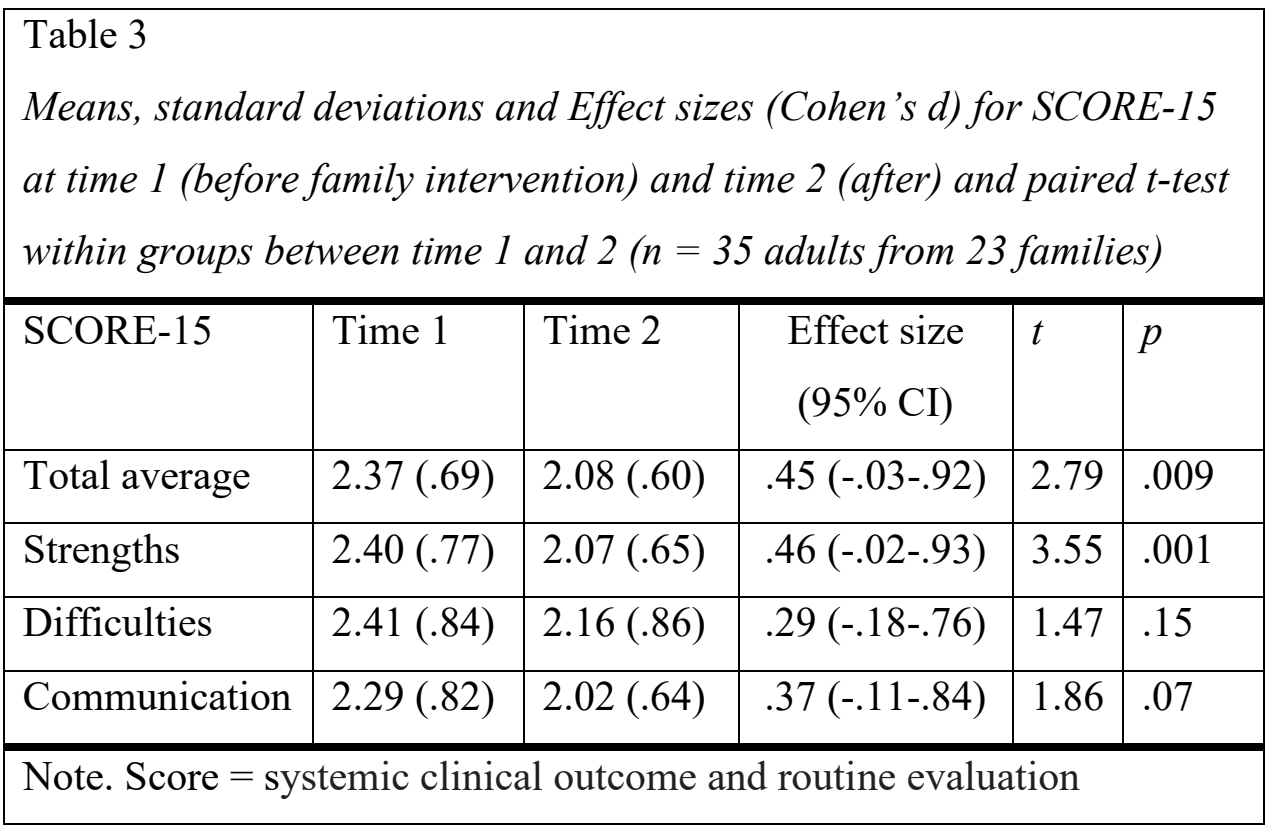




\begin{tabular}{|c|c|c|c|c|c|c|}
\hline $\begin{array}{l}\text { Table } 4 \\
\text { Standardized factor loadings for } S C C \\
\text { family mean, } n=159 \text { ) }\end{array}$ & RE-15 three & -factor $n$ & odel (Clin & ical adults, & $n=215$ & \\
\hline Variable & Stren & gths & Diffi & ulties & Comm & inication \\
\hline & $n=215$ & $n=159$ & $n=215$ & $n=159$ & $n=215$ & $n=159$ \\
\hline $\begin{array}{l}\text { 1. In my family we talk to each } \\
\text { other about things which matter to } \\
\text { us }\end{array}$ & .58 & .65 & & & & \\
\hline $\begin{array}{l}\text { 3. Each of us gets listened to in our } \\
\text { family }\end{array}$ & .58 & .71 & & & & \\
\hline 6. We trust each other & .62 & .61 & & & & \\
\hline $\begin{array}{l}\text { 10. When one of us is upset they get } \\
\text { looked after within the family }\end{array}$ & .63 & .65 & & & & \\
\hline $\begin{array}{l}15 . \text { We are good at finding new } \\
\text { ways to deal with things that are } \\
\text { difficult }\end{array}$ & .53 & .56 & & & & \\
\hline $\begin{array}{l}\text { 5. We find it hard to deal with } \\
\text { everyday problems }\end{array}$ & & & .63 & .56 & & \\
\hline 7. It feels miserable in our family & & & .79 & .80 & & \\
\hline $\begin{array}{l}\text { 9. We seem to go from one crisis to } \\
\text { another in my family }\end{array}$ & & & .72 & .72 & & \\
\hline $\begin{array}{l}\text { 11. Things always seem to go } \\
\text { wrong for my family }\end{array}$ & & & .79 & .79 & & \\
\hline $\begin{array}{l}\text { 14. In my family we blame each } \\
\text { other when things go wrong }\end{array}$ & & & .54 & .57 & & \\
\hline $\begin{array}{l}\text { 2. People often don't tell each other } \\
\text { the truth in my family }\end{array}$ & & & & & .40 & .41 \\
\hline $\begin{array}{l}\text { 4. It feels risky to disagree in our } \\
\text { family }\end{array}$ & & & & & .60 & .53 \\
\hline $\begin{array}{l}\text { 8. When people in my family get } \\
\text { angry they ignore each other on } \\
\text { purpose }\end{array}$ & & & & & .54 & .69 \\
\hline $\begin{array}{l}\text { 12. People in the family are nasty to } \\
\text { each other }\end{array}$ & & & & & .71 & .72 \\
\hline $\begin{array}{l}\text { 13. People in my family interfere } \\
\text { too much in each other's lives }\end{array}$ & & & & & .55 & .56 \\
\hline Interfactor correlation & & trengths & & & ifficultie & \\
\hline & $n=215$ & & $=159$ & $n=215$ & & $=159$ \\
\hline Strengths & - & & - & & & \\
\hline Difficulties & -.58 & & -.62 & - & & - \\
\hline Communication & -.64 & & -.72 & .81 & & .82 \\
\hline
\end{tabular}


Note. SCORE $=$ systemic clinical outcome and routine evaluation. The confirmatory factor analysis was performed both with $(n=215$ and $n=159)$ and without $(n=204$ and $n=151)$ imputed missing items, which only minimally affected results. 